\title{
Sleep Apnea Risk in Subjects With Asthma With or Without Comorbid Rhinitis
}

\author{
Fulvio Braido MD, Ilaria Baiardini PhD, Donato Lacedonia MD, Fabrizio M Facchini MD, \\ Francesco Fanfulla MD, Giorgia Molinengo PhD, and Giorgio W Canonica MD, \\ on behalf of the Italian Society of Respiratory Medicine (SIMeR)
}

\begin{abstract}
BACKGROUND: As many as $80 \%$ of patients with asthma suffer from allergic rhinitis (AR), and rhinitis symptoms are associated with sleep complaints The aim of this cross-sectional study was to assess the prevalence of obstructive sleep apnea syndrome risk in patients with asthma and to explore the association between comorbid rhinitis and obstructive sleep apnea syndrome risk. METHODS: Subjects with asthma were recruited by general practitioners during a control visit. Physicians compiled a questionnaire that assessed the presence of AR according to ARIA (Allergic Rhinitis and Its Impact on Asthma) guidelines and factors influencing the risk of obstructive sleep apnea syndrome (gastroesophageal reflux disease, obesity, smoking). Subjects completed a questionnaire evaluating the presence and severity of AR and the STOP-BANG questionnaire (snoring, tiredness during daytime, observed apnea, high blood pressure, body mass index, age, neck circumference, gender), a validated screening method to identify obstructive sleep apnea syndrome risk. Physicians were blinded to the subjects' questionnaires, ensuring objectivity of the method. RESULTS: The analyses were conducted on 1,941 subjects (males $58 \%$, mean age $48.2 \pm 15.2$ y): 740 with asthma alone and 1,201 with asthma and AR. STOP-BANG revealed that $52.6 \%$ of the subjects were at increased risk of obstructive sleep apnea syndrome: $47.3 \%$ of subjects with asthma alone and 55.9\% of patients with asthma and AR. Rhinitis was associated with a 1.44 times higher odds ratio for having obstructive sleep apnea syndrome risk. Rhinitis duration and severity were associated with obstructive sleep apnea syndrome risk, although the latter deserved greater importance. The results showed that, once a correction for each of these factors was performed, subjects with $A R$ with an odds ratio of 1.99 were reported to be at risk of obstructive sleep apnea syndrome. CONCLUSIONS: The probable increased risk of obstructive sleep apnea syndrome is associated with the concomitant presence of rhinitis, independent of obesity and other contributors to risk of obstructive sleep apnea syndrome. Key words: asthma; allergic rhinitis; obstructive sleep apnea syndrome; risk; sleep apnea; STOP-BANG. [Respir Care 2014;59(12):1851-1856. (O) 2014 Daedalus Enterprises]
\end{abstract}

\section{Introduction}

Almost $80 \%$ of patients with asthma suffer from comorbid rhinitis, ${ }^{1}$ and rhinitis symptoms are associated with

Drs Braido, Baiardini, and Canonica are affiliated with the Allergy and Respiratory Diseases Clinic, Department of Internal Medicine and Medical Specialties, University of Genoa and the Istituto di Ricovero e Cura a Carattere Scientifico Azienda Ospedaliera Universitaria San MartinoInstituto Scientifico Tumori, Genoa, Italy. Dr Lacedonia is affiliated with the Institute of Respiratory Disease, Department of Medical and Surgical Sciences, University of Foggia, Foggia, Italy. Dr Facchini is affiliated sleep complaints. ${ }^{2}$ Furthermore, rhinitis could be considered a risk factor for sleep-disordered breathing ${ }^{3}$ on the basis of physical laws: the Bernoulli principle states that the wider the beginning of the airway, the less the risk of

\footnotetext{
with the UO di Pneumologia, Ospedale di Vittorio Veneto, Vittorio Veneto Treviso, Italy. Dr Fanfulla is affiliated with the Fondazione S Maugeri, Sleep Medicine Center, Istituto di Ricovero e Cura a Carattere Scientifico, Pavia, Italy. Dr Molinengo is affiliated with the Dipartimento di Psicologia, University of Turin, Turin, Italy.
}

The authors have disclosed no conflicts of interest. 
collapse; the narrower the beginning, the greater the risk of risk of collapse. Moreover, the Venturi effect states that air must pass through a small tube faster than through a large tube if the passing volume of air and time are equal. Therefore, the upper airways behave like a Starling resistor: the obstruction at the inlet (ie, the nasal airway) produces collapsing forces that manifest downstream in the collapsible segment, the pharynx. ${ }^{4}$ Clinical observations have shown that patients who report nasal congestion due to allergic rhinitis (AR) are significantly more likely to be habitual snorers and have an increased risk of sleep-disordered breathing. ${ }^{3,5}$ In addition, apneas are longer and more frequent in patients with nasal congestion than in patients without, ${ }^{6}$ and this phenomenon seems to be strictly related to the cross-sectional area of upper airways. ${ }^{7} \mathrm{AR}$ therefore seems involved in snoring, apnea, and hypopnea pathogenesis and can further worsen symptoms of obstructive sleep apnea syndrome. ${ }^{8}$ It has been shown that patients with obstructive sleep apnea syndrome with concomitant AR have significantly higher fatigue, daytime sleepiness, and stress compared with patients with obstructive sleep apnea syndrome without AR. ${ }^{9}$ Cross-sectional ${ }^{10-13}$ and longitudinal ${ }^{14}$ studies found that asthma is associated with both the clinical features of obstructive sleep apnea syndrome ${ }^{11,12,14}$ and diagnosis of obstructive sleep apnea syndrome established by polysomnography. ${ }^{10,13} \mathrm{Bi}-$ directional influences probably exist between the 2 conditions, and obstructive sleep apnea appears to affect asthma control.

Obstructive sleep apnea syndrome was found to be a risk factor for frequent asthma exacerbations in a large population of difficult-to-control patients with asthma. ${ }^{15}$ Additionally, in a cross-sectional survey, obstructive sleep apnea syndrome risk was associated with poorly controlled asthma; indeed, asthmatic patients at high risk of obstructive sleep apnea syndrome have a 3.6 odds ratio to have poorly controlled asthma. Other factors such as obesity, black race, nasal disorders, gastroesophageal reflux disease, and psychiatric diseases are also associated with greater risk of uncontrolled asthma. Nevertheless, multivariate stepwise logistic regression models of uncontrolled patients (also adjusted for the above-mentioned factors) showed that patients with increased obstructive sleep apnea syndrome risk and maintaining an odds ratio of 2.87 are poorly controlled. ${ }^{16}$ More recent evidence showed that obstructive sleep apnea syndrome risk is associated with

Correspondence: Fulvio Braido MD, Allergy and Respiratory Diseases Clinic, DIMI, University of Genoa, IRCCS AOU San Martino-IST, Pad Maragliano, Largo Rosanna Benzi 10, 16132 Genoa, Italy. E-mail: fulvio.braido@unige.it.

DOI: $10.4187 /$ respcare. 03084

\section{QUICK LOOK}

\section{Current knowledge}

Up to $80 \%$ of patients with asthma suffer from allergic rhinitis, which is associated with complaints of sleep disturbances and fatigue. The prevalence of obstructive sleep apnea syndrome in patients with asthma and comorbid rhinitis has not been completely elucidated.

\section{What this paper contributes to our knowledge}

There was an association of increased risk of obstructive sleep apnea syndrome and concomitant presence of rhinitis, independent of obesity and other contributors to risk of obstructive sleep apnea syndrome.

persistent daytime asthma symptoms to an extent that matched or exceeded associations with nighttime asthma symptoms. ${ }^{17}$ In other words, obstructive sleep apnea syndrome is a potential contributor to overall asthma control on a much larger scale and is independent of the other known variables. In summary, AR may worsen obstructive sleep apnea syndrome, and this can negatively influence asthma control. Despite these concerns, there is still a shortage of studies on the association of rhinitis with sleep apnea risk in patients with asthma. The available knowledge is based on the observation of patients with rhinitis and of patients with asthma, whereas no data are available on patients with asthma and comorbid rhinitis. Furthermore, the data on asthma are focused mainly on the effect of obstructive sleep apnea syndrome on disease control, rather than the association between asthma and obstructive sleep apnea syndrome. In addition to the role of obesity, several mechanisms such as overnight increase in parasympathetic tone, nonadrenergic noncholinergic system modulation, and airway inflammation could trigger apnea onset. ${ }^{8}$ The aim of this cross-sectional study was to assess the prevalence of obstructive sleep apnea syndrome risk in subjects with asthma with or without comorbid rhinitis and to explore the association between rhinitis duration and severity and obstructive sleep apnea syndrome risk.

\section{Methods}

A quantitative research program using a questionnaire data collection method was performed; the study design was based on the subjects' recruitment by physicians. The study population included adult subjects with asthma diagnosed by a physician, according to Global Initiative for Asthma ${ }^{18}$ guidelines, while attending a general practitioner's office for scheduled visits. Subjects attending the office for an unscheduled asthma visit, pregnant women, 
and patients with COPD were excluded, but smokers were not. ${ }^{19}$ This study was performed according to good clinical practice standards and the Declaration of Helsinki and was approved by the local ethics committee.

Once written informed consent was obtained, physicians completed a questionnaire consisting of 5 multiple choice questions aimed at assessing the presence of rhinitis and other factors that could influence the risk of obstructive sleep apnea syndrome (ie, gastroesophageal reflux disease, obesity, smoking). Moreover, physicians were asked to indicate whether, according to their point to view, the subject could suffer from obstructive sleep apnea syndrome. Physicians had to provide the classification of severity according to ARIA (Allergic Rhinitis and Its Impact on Asthma) guidelines for subjects suffering from rhinitis. ${ }^{19}$ Subjects were asked to answer a questionnaire including questions about personal characteristics (age, sex, height, weight), presence of 2 or more rhinitis symptoms and their frequency and severity apart from colds, and the presence and frequency of nocturnal awakenings caused by asthma or rhinitis according to ARIA classification. ${ }^{20}$ Moreover, subjects were asked to respond to the STOPBANG questionnaire, a validated screening method to identify individuals at risk of obstructive sleep apnea. ${ }^{21}$ STOPBANG is composed of 8 yes/no questions based upon snoring, tiredness/sleepiness, observed apneas, hypertension, body mass index $>35 \mathrm{~kg} / \mathrm{m}^{2}$, age $>50 \mathrm{y}$, neck circumference $>40 \mathrm{~cm}$, and male gender. A score of 3 or more positive responses is considered increased risk for obstructive sleep apnea syndrome. In fact, a score of $\geq 3$ has shown a high sensitivity for detecting obstructive sleep apnea (93 and 100\% for moderate and severe obstructive sleep apnea, respectively), ${ }^{22}$ and a score of 5-8 has identified patients with high probability of moderate/severe obstructive sleep apnea. ${ }^{23}$ Farney et $\mathrm{al}^{24}$ showed that a score of $>3$ has a $85.1 \%$ probability to identify subjects with obstructive sleep apnea.

Physicians were blinded to the subjects' questionnaires, ensuring objectivity of the method. Because of the observational nature of the study and because the survey was performed in a general practitioner's office, it was established a priori that only subjects for whom rhinitis had been reported by both themselves and the physician in a blinded setting were included.

The study was conducted between March and December 2011 in all Italian regions, and 1,700 general practitioners participating in a continuing medical educational program were involved. In particular, general practitioners were asked to enroll a maximum of 5 consecutive patients with asthma. The recruitment of 3,500 patients with asthma was considered sufficient to explore the study aim. Sample size was estimated considering a $10 \%$ data failure in subjects' and physicians' questionnaire fulfillment, the prevalence of rhinitis in asthma, ${ }^{20}$ an estimated $60 \%$ concor- dance of subjects and physicians in defining concomitant rhinitis presence or absence, and an estimated STOPBANG-defined increased obstructive sleep apnea risk of $27.5 \% .25$

\section{Statistical Analysis}

Analyses were conducted on data from subjects with asthma with or without comorbid rhinitis identified by both physicians and subjects. Baseline variables were summarized as the mean \pm SD for continuous variables and percentiles for categorical variables. A univariate logistic regression model was conducted to test for relationships of increased risk of obstructive sleep apnea syndrome (STOPBANG score $\geq 3$ ) with rhinitis classification according to ARIA guidelines. Multivariate logistic regression models were then performed in subjects with increased risk of obstructive sleep apnea syndrome (defined based on STOPBANG) as the outcome, with stepwise adjustment for factors known to worsen sleep apnea. The aforementioned covariates $(P=.05)$ indicate statistical significance. Analyses were performed using statistical software (SPSS 20, SPSS, Chicago, Illinois).

\section{Results}

Among the 1,700 physicians participating in the continuing medical educational program, 1,678 completed the questionnaire and enrolled at least 2 subjects each. A total of 3,527 subjects who provided informed consent and completed the questionnaire were reached, from an invited population of 3,950 .

Fifty-three subjects were subsequently excluded due to incomplete questionnaires, and the analyses were reduced to a sample of 3,474 subjects. Subjects were considered for the analysis only if their report of presence or absence of concomitant rhinitis was in accordance with the physician's report. The analyses were therefore reduced to a sample of 1,941 subjects: 740 with asthma alone and 1,201 with asthma and comorbid rhinitis. Clinical and demographic data are reported in Table 1.

The majority of the subjects $(75.27 \%)$ reported at least one nocturnal awakening related to a breathing disorder during the month before, of which $40.6 \%$ had 3 or more awakenings per week.

According to the STOP-BANG questionnaire, 1,020 $(52.6 \%)$ subjects were classified as being at increased risk of obstructive sleep apnea syndrome: 350 of 740 (47.3\%) subjects with asthma alone and 670 of $1,021(55.9 \%)$ subjects with asthma and comorbid rhinitis. Subjects' responses to the STOP-BANG questionnaire are reported in Table 2. 
Table 1. Demographic and Clinical Data

\begin{tabular}{lc}
\hline \hline \multicolumn{1}{c}{ Characteristics } & Values \\
\hline Male, \% & 58 \\
Age, mean $\pm \mathrm{SD}, \mathrm{y}$ & $48.2 \pm 15.2$ \\
Neck circumference, mean $\pm \mathrm{SD}, \mathrm{cm}$ & $40.4 \pm 2.2$ \\
Height, mean $\pm \mathrm{SD}, \mathrm{cm}$ & $169.3 \pm 8.7$ \\
Weight, mean $\pm \mathrm{SD}, \mathrm{kg}$ & $74.6 \pm 14.8$ \\
BMI, mean $\pm \mathrm{SD}, \mathrm{kg} / \mathrm{m}^{2}$ & $25.9 \pm 4.6$ \\
Obesity (BMI $>30), \%$ & 17 \\
Gastroesophageal reflux disease, \% & 28 \\
Sleep apnea, \% & 40.55 \\
Active smoker, \% & 19 \\
Rhinitis, \% & 61.88 \\
Rhinitis classification* & \\
$\quad$ Mild intermittent, \% & 35.5 \\
Moderate-severe intermittent, \% & 16.6 \\
Mild persistent, \% & 16.6 \\
$\quad$ Moderate-severe persistent, \% & 31.3 \\
& \\
$n=1,941$ & \\
* Rhinitis classification severity is according to ARIA guidelines. ${ }^{19}$ & \\
BMI = body mass index & \\
\hline
\end{tabular}

Table 2. Subjects' Answers to STOP-BANG Questionnaire

\begin{tabular}{llc}
\hline \hline & & Subjects, $n(\%)$ \\
\hline $\mathrm{S} \quad \begin{array}{l}\text { Do you snore loudly (louder than talking } \\
\text { or loud enough to be heard through } \\
\text { closed doors) }\end{array}$ & $965(50)$ \\
& \\
$\mathrm{T} \quad \begin{array}{l}\text { Do you often feel tired, fatigued, or sleepy } \\
\text { during daytime? }\end{array}$ & $1,208(62)$ \\
$\mathrm{O} \quad$ Has anyone observed you stop breathing \\
$\quad$ during sleep?
\end{tabular}

\section{Association Between Rhinitis and Risk of Obstructive Sleep Apnea Syndrome}

In univariate analyses, rhinitis was associated with a 1.44 times higher odds ratio for having increased obstructive sleep apnea syndrome risk (defined according to STOPBANG). Body mass index $>30 \mathrm{~kg} / \mathrm{m}^{2}$, sex, age, and smoking were also associated with an increased odds ratio for obstructive sleep apnea syndrome risk, whereas gastroesophageal reflux disease was not (Table 3).

A multivariate logistic regression model was performed to assess the presence of an increased risk of obstructive
Table 3. Univariate Associations of High Obstructive Sleep Apnea Risk With Characteristics Known to Predict Obstructive Sleep Apnea

\begin{tabular}{llc}
\hline \hline \multicolumn{1}{c}{ Characteristics } & Odds Ratio (95\% CI) & $P$ \\
\hline Obesity (BMI $>$ 30) & $5.81(4.27-7.92)$ & $<.001$ \\
Gender (male vs female) & $7.8(6.4-9.7)$ & $<.001$ \\
Age (class of 5 y) & $1.42(1.37-1.48)$ & $<.001$ \\
Gastroesophageal reflux disease & $1.18(0.97-1.44)$ & .10 \\
Smoker & $3.15(2.4-4.1)$ & $<.001$ \\
Rhinitis & $1.44(1.17-1.69)$ & $<.001$ \\
& & \\
Defined on the basis of the STOP-BANG questionnaire. & \\
BMI = body mass index & & \\
\end{tabular}

Table 4. Multivariate Logistic Regression Models of Increased Obstructive Sleep Apnea Risk With Adjustment for Factors Known to Worsen Sleep Apnea

\begin{tabular}{lcc}
\hline \hline & Odds Ratio (95\% CI) & $P$ \\
\hline Age, y & $1.08(1.07-1.09)$ & $<.001$ \\
Gender (male vs female) & $8.97(7.42-10.86)$ & $<.001$ \\
Obesity (BMI $>$ 30) & $3.77(2.82-5.04)$ & $<.001$ \\
Gastroesophageal reflux disease & $1.04(0.85-1.27)$ & .70 \\
Smoker & $2.59(2.04-3.30)$ & $<.001$ \\
Rhinitis & $1.37(1.14-1.64)$ & $<.001$
\end{tabular}

Defined on the basis of the STOP-BANG questionnaire. $\mathrm{BMI}=$ body mass index

sleep apnea syndrome, taking into account factors that typically worsen obstructive sleep apnea syndrome. The results showed that, once a correction for each of these factors was performed, subjects with AR with an odds ratio of 1.99 were reported to be at risk of obstructive sleep apnea syndrome (Table 4).

\section{Discussion}

Data from a large sample of subjects with asthma showed that increased risk of obstructive sleep apnea syndrome is associated with the concomitant presence of rhinitis. This relationship emerged independently of obesity and other contributors to risk of obstructive sleep apnea syndrome. Although this cross-sectional study cannot prove causal relationships, our data and analyses support available evidence for the relationship between obstructive sleep apnea syndrome and rhinitis. This finding further emphasizes the clinical problem in a cohort of subjects, such as patients with asthma, in which the prevalence of rhinitis is very high. Rhinitis is a well-known asthma trigger, but also could be involved in the pathogenesis of sleep breathing disorders, such as obstructive sleep apnea syndrome. 
Based on analysis of a large population sample, according to the STOP-BANG score, subjects with comorbid rhinitis exhibit an increased risk of obstructive sleep apnea syndrome (odd ratios of 1.37) compared with those subjects without it. A multivariate logistic regression model of increased risk of obstructive sleep apnea with adjustment for well-known worsening factors of sleep apnea confirmed that when age, sex, smoking, obesity, and gastroesophageal reflux disease were sequentially excluded, rhinitis remained associated with an increased risk of obstructive sleep apnea syndrome. These results suggest an intriguing relationship between rhinitis and obstructive sleep apnea syndrome. However, they need to be further confirmed using a well-defined population of patients with asthma and with obstructive sleep apnea syndrome diagnosis confirmed or excluded by polysomnography.

As our data suggest, there is a likely substantial burden of unrecognized and untreated obstructive sleep apnea in patients with asthma. This is a population that could benefit considerably from having their sleep disorder addressed, as other data and analyses have also described. ${ }^{26,27}$

Limitations of our study include the use of a questionnaire-based approach since polysomnography seemed to be prohibitively expensive for this cross-sectional study given the sample size and subjects' recruitment setting. However, in a large sample of sleep subjects, the STOPBANG method demonstrated high internal validity and good sensitivity. ${ }^{25}$ Furthermore, this instrument has high diagnostic value in comparison with other sleep apnea screening instruments. ${ }^{21}$

\section{Conclusions}

In summary, this study shows that rhinitis is associated with the probable increased risk of obstructive sleep apnea syndrome in patients with asthma. Clinicians should consider rhinitis as a potential reason for poor asthma control, but also as an associated factor of obstructive sleep apnea syndrome, which is a cause itself of increased asthma symptoms. Prospective studies with objective sleep and airway assessment of these putative mechanistic links are recommended to enhance understanding and identify means to intervene in asthma with comorbid rhinitis and obstructive sleep apnea syndrome.

\section{ACKNOWLEDGMENTS}

The study was endorsed by the Italian Society of Respiratory Medicine. We thank the Associazione Ricerca Malattie Immunologiche e Allergiche and the Associazione Pazienti Disturbi Respiratori nel Sonno and acknowledge Dr Marianna Bruzzone for linguistic assistance with the manuscript.
Italian Society of Respiratory Medicine Investigators:

University of Genoa, Genoa, Italy: Vito Brusasco MD, Respiratory Physiopathology Unit, Department of Internal Medicine; Manlio Milanese MD, Department of Pharmacy, Pharmacology and Toxicology Section, and Center of Excellence for Biomedical Research.

Università degli Studi di Milano, Milan, Italy: Stefano Centanni MD, UOC Pneumologia, Ospedale San Paolo.

Università di Pavia, Pavia, Italy: Angelo Corsico MD, Dipartimento di Medicina Molecolare, Fondazione Istituto di Ricovero e Cura a Carattere Scientifico Policlinico San Matteo.

University Hospital, Messina, Italy: Giuseppe Girbino MD, Respiratory Department.

Ospedale San Paolo, Milan, Italy: Fabiano Di Marco MD, Clinica di Malattie Respiratorie-UOC Pneumologia.

University of Catania, Catania, Italy: Giuseppe Di Maria MD, School of Specialization in Respiratory Diseases.

Santa Corona Hospital, Pietra Ligure, Savona, Italy: Carlo Mereu MD, Pulmonology Department.

Università degli Studi di Milano, Milan, Italy: Pierachille Santus MD, Pneumologia Riabilitativa-Fondazione Salvatore Maugeri, Istituto Scientifico di Riabilitazione di Milano (Istituto di Ricovero e Cura a Carattere Scientifico).

University of Palermo, Palermo, Italy: Nicola Scichilone MD, Dipartimento Biomedico di Medicina Interna e Specialistica, "Villa SofiaCervello" Hospital.

Citta della Salute e della Scienza di Torino, Turin, Italy: Paolo Solidoro MD, Unit of Pneumology, Cardiovascular and Thoracic Department, San Giovanni Battista Molinette Hospital.

\section{REFERENCES}

1. Navarro A, Valero A, Juliá B, Quirce S. Coexistence of asthma and allergic rhinitis in adult patients attending allergy clinics: ONEAIR study. J Investig Allergol Clin Immunol 2008;18(4):233-238.

2. Léger D, Annesi-Maesano I, Carat F, Rugina M, Chanal I, Pribil C. Allergic rhinitis and its consequences on quality of sleep. Arch Intern Med 2006;166(16):1744-1748.

3. Young T, Finn L, Palta M. Chronic nasal congestion at night is a risk factor for snoring in a population-based cohort study. Arch Intern Med 2001;161(12):1514-1519.

4. Georgalas C. The role of the nose in snoring and obstructive sleep apnoea: an update. Eur Arch Otorhinolaryngol 2011;268(9):1365-1373.

5. Young T, Finn L, Kim H. Nasal obstruction as a risk factor for sleep-disordered breathing. The University of Wisconsin Sleep and Respiratory Research Group. J Allergy Clin Immunol 1997;99(2): S757-S762.

6. McNicholas WT, Tarlo S, Cole P, Zamel N, Rutherford R, Griffin D, Phillipson EA. Obstructive apneas during sleep in patients with seasonal allergic rhinitis. Am Rev Respir Dis 1982;126(4):625-628.

7. Houser SM, Mamikoglu B, Aquino BF, Moinuddin R, Corey JP. Acoustic rhinometry findings in patients with mild sleep apnea. Otolaryngol Head Neck Surg 2002;126(5):475-480.

8. Alkhalil M, Schulman E, Getsy J. Obstructive sleep apnea syndrome and asthma: what are the links? J Clin Sleep Med 2009;5(1):71-78.

9. Park CE, Shin SY, Lee KH, Cho JS, Kim SW. The effect of allergic rhinitis on the degree of stress, fatigue and quality of life in OSA patients. Eur Arch Otorhinolaryngol 2012;269(9):2061-2064.

10. Yigla M, Tov N, Solomonov A, Rubin AH, Harlev D. Difficult-tocontrol asthma and obstructive sleep apnea. J Asthma 2003;40(8):865871.

11. Teodorescu M, Consens FB, Bria WF, Coffey MJ, McMorris MS, Weatherwax KJ, et al. Correlates of daytime sleepiness in patients with asthma. Sleep Med 2006;7(8):607-613. 


\section{Sleep Apnea Risk in Subjects With Asthma}

12. Auckley D, Moallem M, Shaman Z, Mustafa M. Findings of a Berlin questionnaire survey: comparison between patients seen in an asthma clinic versus internal medicine clinic. Sleep Med 2008;9(5):494-499.

13. Julien JY, Martin JG, Ernst P, Olivenstein R, Hamid Q, Lemière C, et al. Prevalence of obstructive sleep apnea-hypopnea in severe versus moderate asthma. J Allergy Clin Immunol 2009;124(2):371-376.

14. Knuiman M, James A, Divitini M, Bartholomew H. Longitudinal study of risk factors for habitual snoring in a general adult population: the Busselton Health Study. Chest 2006;130(6):1779-1783.

15. ten Brinke A, Sterk PJ, Masclee AA, Spinhoven P, Schmidt JT, Zwinderman $\mathrm{AH}$, et al. Risk factors of frequent exacerbations in difficult-to-treat asthma. Eur Respir J 2005;26(5):812-818.

16. Teodorescu M, Polomis DA, Hall SV, Teodorescu MC, Gangnon RE, Peterson AG, et al. Association of obstructive sleep apnea risk with asthma control in adults. Chest 2010;138(3):543-550.

17. Teodorescu M, Polomis DA, Teodorescu MC, Gangnon RE, Peterson AG, Consens FB, et al. Association of obstructive sleep apnea risk or diagnosis with daytime asthma in adults. J Asthma 2012;49(6):620-628.

18. Bateman ED, Hurd SS, Barnes PJ, Bousquet J, Drazen JM, FitzGerald $\mathrm{M}$, et al. Global strategy for asthma management and prevention: GINA executive summary. Eur Respir J 2008;31(1):143-178.

19. Bousquet PJ, Cropet C, Klossek JM, Allaf B, Neukirch F, Bousquet J. Effects of smoking on symptoms of allergic rhinitis. Ann Allergy Asthma Immunol 2009;103(3):195-200.

20. Bousquet J, Khaltaev N, Cruz AA, Denburg J, Fokkens WJ, Togias A, et al. Allergic Rhinitis and its Impact on Asthma (ARIA) 2008 update (in collaboration with the World Health Organization, GA(2)LEN and AllerGen). Allergy 2008;63(Suppl):8-160.

21. Abrishami A, Khajehdehi A, Chung. A systematic review of screening questionnaires for obstructive sleep apnea. Can J Anaesth 2010; 57(5):423-438

22. Chung F, Yegneswaran B, Liao P, Chung SA, Vairavanathan S, Islam S, et al. Validation of the Berlin questionnaire and American Society of Anesthesiologists checklist as screening tools for obstructive sleep apnea in surgical patients. Anesthesiology 2008;108(5): 822-830.

23. Chung F, Subramanyam R, Liao P, Sasaki E, Shapiro C, Sun Y. High STOP-Bang score indicates a high probability of obstructive sleep apnoea. Br J Anaesth 2012;108(5):768-775.

24. Farney RJ, Walker BS, Farney RM, Snow GL, Walker JM. The STOP-Bang equivalent model and prediction of severity of obstructive sleep apnea: relation to polysomnographic measurements of the apnea/hypopnea index. J Clin Sleep Med 2011;7(5):459-465

25. Chung F, Yegneswaran B, Liao P, Chung SA, Vairavanathan S, Islam S, et al. STOP questionnaire: a tool to screen patients for obstructive sleep apnea. Anesthesiology 2008;108(5):812-821.

26. Chan CS, Woolcock AJ, Sullivan CE. Nocturnal asthma: role of snoring and obstructive sleep apnea. Am Rev Respir Dis 1988;137(6): 1502-1504.

27. Lafond $\mathrm{C}$, Sériès $\mathrm{F}$, Lemière $\mathrm{C}$. Impact of CPAP on asthmatic patients with obstructive sleep apnoea. Eur Respir J 2007;29(2):307-311. 